\title{
Prevalência de Parasitoses em Idosos residentes e trabalhadores de Instituições de Longa Permanência na Região Metropolitana de Porto Alegre e na Região Serrana do Rio Grande do Sul ${ }^{\#}$
}

Prevalence of Parasitosis in Elderly residents and Workers of Institutions of Long Permanence in the Metropolitan Region of Porto Alegre and the Mountain Region of Rio Grande do Sul

\author{
Anne Brandolt Larré* \\ Camila Duarte Bürgie** \\ Paula Engroff*** \\ Geraldo Attilo De Carli****
}

\begin{abstract}
Resumo
Apesar dos enteroparasitos comprometerem o estado nutricional de idosos e a qualidade da saúde em geral, estudos abordando ocorrência e prevenção de parasitas intestinais, nesse segmento populacional, são escassos. O escopo do presente estudo é analisar o perfil de enteroparasitos nos idosos institucionalizados e funcionários desses lares geriátricos, correlacionando-os com variáveis sóciodemográficas, econômicas, hábitos de higiene e sintomatologia. Trata-se de um estudo transversal realizado em Instituições de Longa Permanência na Região Metropolitana de Porto Alegre e na Região Serrana do Rio Grande do Sul. Através da aplicação de questionários e coletas de amostras fecais, 12 lares geriátricos foram pesquisados e 200 amostras de fezes de idosos, com média de idade de 79,4 49,5 anos, foram analisadas pelo exame parasitológico de fezes (EPF). Também coletamos amostras de 26 funcionários (com faixa etária entre 19 a 60 anos). Verificou-se prevalência de 4,0\% de enteroparasitoses em relação aos idosos, sendo maior positividade encontrada nas mulheres $4,8 \%(n=7)$, enquanto nos homens $1,8 \%$ ( $n=1)$. Dos quais, $75,0 \%$ $(\mathrm{n}=6)$ correspondiam a Endolimax nana e 25,0\% (n=2) a Entamoeba coli. Identificou-se nos funcionários, prevalência de 19,2\%, dos quais $60,0 \%(n=3)$ correspondiam a $E$. nana, 20,0\% $(n=1)$ a $E$. coli e 20,0\% (n=1) de biparasitismo. Evidenciamos condições peculiares nestas duas regiões gaúchas. Pois o envelhecimento traz alguns desafios para a sociedade, exigindo implantação e efetivação de políticas públicas sociais e de saúde condizentes com a realidade. Assim, melhoraríamos a qualidade de vida destes idosos e trabalhadores em relação as suas variáveis sociodemográficas, econômicas, hábitos de higiene e sintomatologia.
\end{abstract}

Palavras-chave: Enteroparasitoses. Idosos. Trabalhadores. Instituições de Longa Permanência.

\begin{abstract}
Despite enteroparasites compromise the nutritional status of the elderly and health care quality in general, studies addressing the occurrence and prevention of intestinal parasites in this population segment are scarce. The scope of this study is to analyze the enteroparasites profile in the elderly and employees working in long-term institutions, correlating them to sociodemographic and economic variables, hygiene habits and symptoms. It is a cross-sectional study in long-term institutions in the Metropolitan Area of Porto Alegre and in the Mountain Region of Rio Grande do Sul. Through the use of questionnaires and collection of stool samples, 12 long-term institutions were searched and 200 stool samples of feces, with an average of $79.4 \pm 9.5$ years old, were analyzed by Parasitological Examination of Stools. In addition, we collect 26 samples from employees (aged between 19-60 years old). It was found a prevalence of $4.0 \%$ of intestinal parasites in relation to the elderly, the higher positivity found in women $4.8 \%(n=7)$, while in men $1.8 \%(n=1)$. Of which $75.0 \%(n=6)$ corresponded to Endolimax nana and $25.0 \%(n=2)$ Entamoeba coli. It was identified in employees, prevalence of $19.2 \%$, of which $60.0 \%(\mathrm{n}=3)$ accounted for $E$. nana, 20.0\% $(\mathrm{n}=1) E$. coli and $20.0 \%(n=1)$ of hyperparasitism. Both regions show peculiar conditions, aging brings some challenges to society, demanding the implementation and effectiveness of social policies of public health congruent to the current reality to improve the life quality for elderly and workers regarding their sociodemographic variables, economic, hygienic habits and symptomatology.
\end{abstract}

Keywords: Enteroparasitosis. Elderly. Workers. Long-term Institutions.

DOI: 10.15343/0104-7809.201539018491

\# Manuscrito resultante da tese de mestrado de Anne Brandolt Larré (autora), intitulado: prevalência de infecções por enteroparasitos na população de idosos residentes em instituições de longa permanência na região metropolitana de porto alegre e na serra do rio grande do sul, 2014 - (PUCRS).

* Instituto de Geriatria e Gerontologia, PUCRS, Porto Alegre, Brasil. e-mail: anne.larre@bol.com.br

** Faculdade de Farmácia, PUCRS, Porto Alegre, Brasil. E-mail: caburgie@gmail.com

*** Instituto de Geriatria e Gerontologia, PUCRS, Porto Alegre, Brasil. E-mail: paula_puc@yahoo.com.br

**** Instituto de Geriatria e Gerontologia, PUCRS, Porto Alegre, Brasil. E-mail: gdecarli@ portoweb.com.br

Os autores declaram não haver conflitos de interesse. 


\section{INTRODUÇÃO}

Nos países em desenvolvimento, como no Brasil, avanços científicos e tecnológicos, adoção de um modo de vida mais saudável, atitudes preventivas em relação às doenças, melhores condições de higiene e outros fatores associados, tiveram como resultado um considerável aumento na expectativa de vida e no consequente crescimento da população idosa. ${ }^{1}$ Estudos abordando a ocorrência e prevenção de parasitos intestinais, na população idosa são escassos, devido a pequena importância atribuída a estes problemas. Entretanto, os enteroparasitos contribuem no comprometimento do estado nutricional e da saúde deste segmento populacional.

As parasitoses intestinais são frequentes em locais onde existem baixas condições socioeconômicas. ${ }^{2}$ Os idosos residentes em Instituições de Longa Permanência (ILPIs), apresentam maior grau de dependência e de doenças clínicas, em relação aos que vivem na comunidade, apresentando maior risco de adquirir doenças infecto parasitárias. ${ }^{3}$ Comportamento deficitário de higiene, mãos contaminadas, alimentos e água, podem transmitir doenças parasitárias. Demonstrando, assim, que as Instituições de Longa Permanência, são ambientes propícios para ocorrência dessas infecções, devido ao contato entre idosos, enfermeiros, técnicos de enfermagem, cuidadores e manipuladores de alimentos. ${ }^{4}$ Atitudes simples de higiene e cuidados pessoais facilmente aprendidos e inseridos no cotidiano e aos hábitos das pessoas, podem evitar a transmissão de doenças parasitárias, desde que seja demonstrada a sua importância à comunidade. ${ }^{5}$

O objetivo desta pesquisa foi analisar o perfil de enteroparasitoses nos idosos institucionalizados e funcionários que trabalham nos lares geriátricos, correlacionando com as variáveis sociodemográficas, econômicas, hábitos de higiene e sintomatologia.

\section{MÉTODO}

Estudo transversal realizado durante o período de Janeiro a Agosto de 2013, em 12 Instituições de Longa Permanência na Região
Metropolitana de Porto Alegre (nas cidades de Novo Hamburgo e São Leopoldo) - colonizadas especialmente por alemães e na Região Serrana do Rio Grande do Sul (nas cidades de Bento Gonçalves e Caxias do Sul) - colonizadas especialmente por italianos. Os lares foram escolhidos para o estudo de forma aleatória, considerando que para cada cidade, deveriam ser inclusos ao menos uma instituição pública e uma particular. O responsável legal de cada instituição assinou um termo autorizando que a pesquisa fosse realizada.

Participaram da pesquisa indivíduos com idade igual ou superior a 60 anos e funcionários das Instituições de Longa Permanência que tinham interesse em aderir ao estudo. Os critérios de exclusão do estudo foram idosos que não possuíam condições de responder ao questionário, de coletar as amostras de fezes, seja por debilidade física ou mental e que faziam uso de laxantes. O total de idosos residentes nas Instituições de Longa Permanência era de 378, sendo que 91 (24\%) não participaram por algum critério de exclusão e 87 (23\%) não aceitaram participar da pesquisa.

Todos os que foram submetidos ao estudo assinaram o Termo de Consentimento Livre e Esclarecido (TCLE), em seguida, os idosos e os trabaIhadores foram entrevistados, respectivamente. O instrumento aplicado foi um questionário contendo informações sociodemográficas, sobre hábitos de higiene, e inquérito coprológico.

Quanto à coleta de amostra do material fecal, os idosos e os funcionários das Instituições de Longa Permanência foram previamente orientados, recebendo as instruções impressas para facilitar sua realização, além de um pote com tampa de rosca para armazenamento dos espécimes fecais e uma espátula de madeira para auxiliar no recolhimento da porção de fezes.

As fezes foram analisadas através do exame parasitológico de fezes (EPF) e a amostra fecal foi submetida ao diagnóstico laboratorial na forma de espécime fresco no Laboratório de Bioquímica, Genética Molecular e Parasitologia do Instituto de Geriatria e Gerontologia da PUCRS - LABGEMP. Foi avaliada a presença de 
enteroparasitos nas fezes dos idosos residentes nas Instituições de Longa Permanência de cada região (Metropolitana de Porto Alegre e Serrana do Rio Grande do Sul), através de técnicas e métodos de avaliação, tanto macroscopicamente (verificando consistência, presença ou ausência de sangue, muco, proglotes de vermes adultos ou outras condições anormais), quanto microscopicamente, através dos métodos e técnicas de avaliação: técnica de sedimentação espontânea de Lutz ou Hoffman, Pons e Janerer, e Método de Baermann-Moraes (para isolamento de larvas de nematóides). ${ }^{8}$

A análise estatística foi realizada pelo programa SPSS versão 17.0. Os resultados foram expressos por frequências, média e desvio padrão. A associação entre as variáveis foi realizada pelo teste do Qui-quadrado. Nas associações que apresentavam uma linearidade, foi utilizado o teste de tendência linear, considerando significativo $p<0,05$.

Essa pesquisa foi aprovada pelo Comitê de Ética em Pesquisa da PUCRS, sob o número de protocolo 147.597.

\section{RESULTADOS}

Foram analisados 12 lares geriátricos nas cidades de Novo Hamburgo 3, São Leopoldo 3, Bento Gonçalves 2 e Caxias do Sul 4. Foram coletadas 200 amostras de fezes de 200 idosos, $145(72,5 \%)$ mulheres e 55 (27,5\%) homens, com média de idade de 79,4 \pm 9,5 anos, sendo que $49,5 \%$ residiam em lares geriátricos na Região Metropolitana de Porto Alegre (nas cidades de Novo Hamburgo e São Leopoldo) e 50,5\% na Região Serrana do Rio Grande do Sul (nas cidades de Bento Gonçalves e Caxias do Sul). Além de 26 funcionários, (entre eles enfermeiros, técnicos de enfermagem, cuidadores, trabalhadores da parte administrativa dos lares e manipuladores de alimentos), com faixa etária entre 19 a 60 anos. Verificou-se uma prevalência de 4,0\% em relação aos idosos acometidos por protozoários intestinais, sendo a maior prevalência encontrada nas mulheres $4,8 \%(n=7)$, enquanto nos homens correspondeu a $1,8 \%(\mathrm{n}=1)$ de $E$. nana. Dessas amostras
$75,0 \%(n=6)$ correspondiam a $E$. nana e $25,0 \%$ $(\mathrm{n}=2)$ E. coli. A taxa de infecção por enteroparasitos foi maior em idosos que possuíam entre 80 e 89 anos (6,8\%), quando relacionados com as outras faixas etárias, porém, sem diferença estatisticamente significativa, utilizando o Valor de $P$ calculado pelo teste de tendência Linear do Qui-quadrado, dados também observados em relação à escolaridade. Observou-se uma maior taxa de positividade de enteroparasitos nas instituições particulares com 4,7\% (n=6), do que nas públicas onde o resultado foi de $2,8 \%(n=2)$, em relação aos tipos de Instituições de Longa Permanência estudadas. Porém, sem diferença significativa, utilizando o Valor de $P$ calculado pelo teste de Pearson do Qui-quadrado, (Tabela 1).

Com relação aos idosos institucionalizados nas cidades pesquisadas, em Bento Gonçalves, não houve infecção por enteroparasitos, em Caxias do Sul 2,0\% de prevalência, em Novo Hamburgo 1,0\% de positividade e em São Leopoldo $5,0 \%$ de prevalência (Tabela 2 ).

Entretanto, quanto aos hábitos alimentares, os idosos que relataram comer mais saladas, verduras e frutas tiveram um índice de prevalência maior $(25,0 \%)$ e significativo estatisticamente $(p=0,008)$, em relação aos que comiam de tudo e aos que ingeriam apenas carnes, pães e massas.

O modo de lavar as saladas, verduras e frutas, bem como, o hábito de lavar as mãos após ir ao banheiro, não mostrou ter diferença estatisticamente significativa em relação à taxa de infecção por enteroparasitos (Tabela 3).

Já idosos que não cortavam as unhas, tiveram $20,0 \%$ de prevalência, mostrando uma tendência estatística $(p=0,064)$. Sugerindo que o modo de transmissão deve ter ocorrido de maneira conhecida como auto-infecção externa ou direta: do ânus para cavidade oral, por meio dos dedos. ${ }^{7}$ Desta forma, devemos orientar constantemente, quanto a hábitos de higiene pessoais, principalmente, no que diz respeito à lavagem das mãos antes das refeições, antes de quaisquer tipos de manipulação de alimentos, água e após utilização de instalações sanitárias. 
Tabela 1. Distribuição sociodemográfica relacionada à prevalência de enteroparasitos, realizada durante o período de Janeiro a Agosto de 2013.

\begin{tabular}{|c|c|c|c|}
\hline Variável & $\begin{array}{c}\text { População Analisada } \\
\text { N (\%) }\end{array}$ & $\begin{array}{c}\text { Prevalência Parasitoses } \\
\text { N (\%) }\end{array}$ & $\boldsymbol{P}$ \\
\hline \multicolumn{4}{|c|}{ DADOS SOCIODEMOGRÁFICOS } \\
\hline \multicolumn{4}{|l|}{ Sexo } \\
\hline Feminino & $145(72,5)$ & $7(4,8)$ & $0,332 *$ \\
\hline Masculino & $55(27,5)$ & $1(1,8)$ & \\
\hline \multicolumn{4}{|l|}{ Faixa Etária (idade) } \\
\hline 60-69 anos & $36(18,0)$ & $1(2,8)$ & \\
\hline 70-79 anos & $57(28,5)$ & $2(3,5)$ & $0,953^{\#}$ \\
\hline 80-89 anos & $74(37,0)$ & $5(6,8)$ & \\
\hline 90 anos ou mais & $33(16,5)$ & $0(0,0)$ & \\
\hline \multicolumn{4}{|l|}{ Escolaridade } \\
\hline Sem Escolaridade & $58(29,0)$ & $1(1,7)$ & \\
\hline $1^{\circ}$ Grau Completo & $24(12,0)$ & $0(0,0)$ & $0,155^{\#}$ \\
\hline $1^{\circ}$ Grau Incompleto & $101(50,5)$ & $6(5,9)$ & \\
\hline $2^{\circ} \mathrm{Grau}$ ou mais & $17(8,5)$ & $1(5,9)$ & \\
\hline \multicolumn{4}{|l|}{ Tipo de Instituições } \\
\hline Particulares & $128(64)$ & $6(4,7)$ & $0,508^{*}$ \\
\hline Públicas & $72(36)$ & $2(2,8)$ & \\
\hline
\end{tabular}

*Valor de $P$ calculado pelo teste de tendência Linear do Qui-quadrado;

* Valor de $P$ calculado pelo teste de Pearson do Qui-quadrado.

Tabela 2. Distribuição de dados relacionados às cidades e regiões analisadas de acordo com a prevalência de enteroparasitos, realizada durante o período de Janeiro a Agosto de 2013.

\begin{tabular}{|c|c|c|c|}
\hline Variável & $\begin{array}{c}\text { População Analisada } \\
\text { N (\%) }\end{array}$ & $\begin{array}{c}\text { Prevalência Parasitoses } \\
\text { N (\%) }\end{array}$ & $P$ \\
\hline \multicolumn{4}{|l|}{ DADOS SOCIODEMOGRÁFICOS } \\
\hline Novo Hamburgo & $59(29,5)$ & $1(1,7)$ & \\
\hline São Leopoldo & $40(20,0)$ & $5(12,5)$ & \\
\hline \multirow{2}{*}{ Total Região Metropolitana Porto Alegre } & $99(49,5)$ & $6(6,1)$ & \\
\hline & & & $0,022^{*}$ \\
\hline Bento Gonçalves & $18(9,0)$ & $0(0,0)$ & \\
\hline Caxias do Sul & $83(41,5)$ & $2(2,4)$ & \\
\hline Total da Região Serrana (RS) & $101(50,5)$ & $2(2,0)$ & \\
\hline TOTAL & 200 & 4,0 & \\
\hline
\end{tabular}

* Valor de $P$ calculado pelo teste de Pearson do Qui-quadrado;

(RS) - Estado do Rio Grande do Sul. 
Tabela 3. Prevalência de enteroparasitos, distribuição de dados de higiene em 200 Idosos Residentes em 12 Instituições de Longa Permanência na Região Metropolitana de Porto Alegre e na Região Serrana do Rio Grande do Sul, realizada durante o período de Janeiro a Agosto de 2013.

\begin{tabular}{lccc}
\hline Variável & $\begin{array}{c}\text { População Analisada } \\
\text { N (\%) }\end{array}$ & $\begin{array}{c}\text { Prevalência de } \\
\text { Parasitoses } \\
\text { N (\%) }\end{array}$ & $P$ \\
\hline HÁBITOS DE HIGIENE & & & \\
\hline Lavagem de Saladas, Verduras e Frutas & $107(53,5)$ & $4(3,7)$ & $0,900^{*}$ \\
\hline Água Sanitária & $56(28,0)$ & $3(5,4)$ & $0(0,0)$ \\
Apenas com água & $6(3,0)$ & $1(3,2)$ & \\
Água e Vinagre & $31(15,5)$ & & $0,064^{*}$ \\
Hipocal & & $1(20,0)$ & $7(3,6)$ \\
\hline Corte de Unhas & $5(2,5)$ & & \\
\hline Não & $195(97,5)$ & & \\
Sim & & & \\
\hline
\end{tabular}

* Valor de $P$ calculado pelo teste de Pearson do Qui-quadrado.

\# Hypocal Tablete: Produto desenvolvido para aplicações em desinfecção de água para consumo humano, animal, industrial e agroindustrial.

Com relação à sintomatologia, as variáveis: constipação, desconforto abdominal, perda de apetite, náusea, vômito, emagrecimento e prurido anal, não tiveram diferença significativa.

Quanto aos trabalhadores das Instituições de Longa Permanência, 96,2\% ( $\mathrm{n}=25)$ eram mulheres e $3,8 \%(n=1)$ homem, com média de idade de 37,3 $\pm 10,0$ anos. Verificou-se uma prevalência de $19,2 \%$, sendo a maior frequência encontrada nas mulheres $20,0 \%(n=5)$. Onde $60,0 \%$ $(\mathrm{n}=3)$ correspondiam a $E$. nana, 20,0\% $(\mathrm{n}=1)$ E. coli e 20,0\% $(\mathrm{n}=1)$ biparasitismo (E. nana e E. coli), não sendo observados casos de poliparasitismo. Em Novo Hamburgo, São Leopoldo e Caxias do Sul, tivemos colaboração de 0,0\% $(n=9), 27,3 \%(n=11)$ e 33,3\% $(n=6)$ funcionários, respectivamente. Em Bento Gonçalves não houve participação deste grupo.

A taxa de infecção por enteroparasitos mostrou uma tendência em funcionários das (ILPIs) Instituições de Longa Permanência que possuíam entre 40 anos ou mais (36,4\%), quando comparados com as outras faixas etárias $(p=0,059)$, utilizou-se o Valor de $P$ calculado pelo teste de tendência Linear do Qui-quadrado. Em relação a menor escolaridade: primeiro grau incompleto, observamos uma maior prevalência de enteroparasitoses $(42,9 \%)(p=0,020)$, utilizando o Valor de $P$ calculado pelo teste de Pearson do Qui-quadrado.

Com relação à sintomatologia, as variáveis: constipação, desconforto abdominal, perda de apetite, náusea, vômito, emagrecimento e prurido anal, não tiveram diferença significativa. Problemas socioeconômicos, de higiene e educação sanitária dos funcionários, levam a crer que esses se tornam mais expostos a infecções e contaminações, quando saem das instituições. Provavelmente, por não manterem as mesmas normas básicas de educação sanitária, exigidas e utilizadas em seus ambientes de trabalho.

\section{DISCUSSÃO}

A prevalência de parasitoses intestinais encontrada nesta pesquisa foi um pouco inferior quando comparada a outros estudos realizados no Estado do Rio Grande do Sul, como aquele de Ely et al. ${ }^{3}$ (2010), com prevalência de $12,9 \%$, tanto para os idosos institucionalizados quanto para os idosos que frequentavam o ambulatório geriátrico, na cidade de Porto Alegre. Devido à falta de dados sobre a incidência de 
enteroparasitoses, no estado do Rio Grande do Sul, torna-se muito difícil estimar precisamente a repercussão do parasitismo intestinal sobre a saúde e a economia da população de idosos e trabalhadores estudados na Região Metropolitana de Porto Alegre e na Região Serrana Gaúcha. ${ }^{3}$

Espécies intestinais como E. nana e E. coli, embora sejam consideradas não patogênicas, sendo comensais no intestino humano, é importante salientar que os índices encontrados, uma vez que esse é um parâmetro para medir o grau de contaminação fecal a que os indivíduos estão expostos. ${ }^{9}$ Sugerindo uma exposição a contaminação, ocorrendo de forma mais acentuada fora dos lares geriátricos. Essas espécies apresentam os mesmos mecanismos de transmissão de outros protozoários, como Entamoeba histolytica/dispar e Giardia lamblia, podendo servir como bons indicadores das condições sócio-sanitárias. Além disso, podem sugerir a presença de comportamentos relacionados à falta de higiene como lavagem inadequada de mãos, água e ocorrência de alimentos contaminados. ${ }^{10}$

Esta pesquisa mostrou que a prevalência de enteroparasitos foi maior nas mulheres, coincidindo com achados em outros estudos. Na literatura sobre envelhecimento, tornou-se comum este fato, principalmente nas idosas institucionalizadas que possivelmente atingem uma maior sobrevida, devido aos cuidados extras referentes à prevenção das doenças e consequentemente maiores expectativa de vida sobre os homens. Confirma-se assim o fenômeno do aumento do sexo feminino na velhice. A prevalência na faixa etária superior a 80 anos, já era esperada, pois o aumento da longevidade da população brasileira vem alterando e elevando a proporção deste estrato da população. ${ }^{11}$

Já em relação ao tipo de instituição estudado, foi visto que nas particulares obtivemos uma maior prevalência. Isso se deve ao fato de que as Instituições de Longa Permanência públicas da Região Metropolitana e da Serrana do (RS) Rio Grande do Sul encontravam-se muito mais estruturadas e organizadas visto que eram sustentadas em sua maioria, pelo município, em questão. Enquanto, os lares particulares, além de pouco pessoal preparado para atender este tipo de demanda de serviço, tinham em sua maioria, condições menos favoráveis tanto de higiene quanto de funcionamento em geral. Esses estudos comprovam que a falta de higiene e de estrutura é fator para maior prevalência de parasitoses. . $^{3,2,13,14}$

Quantos aos índices de prevalência nas cidades estudadas, a ausência de positividade em Bento Gonçalves justifica-se por ser a cidade referência em qualificação do abastecimento de água e tratamento do esgoto com investimentos de saneamento na Região Serrana do RS, gerando um aumento significativo de $40 \%$ de beneficiados. ${ }^{15,16}$ Em São Leopoldo e Novo Hamburgo, cidades banhadas pelo Rio dos Sinos, área de maior concentração de curtumes do estado gaúcho, ocorre um predomínio de utilização de fossa séptica e quase $70 \%$ do esgoto produzido vai direto para o rio, apresentando um dos maiores déficits de atendimento urbano com serviços de esgoto, com redução na capacidade da infraestrutura de rede coletora de esgoto e de tratamento, mostrando-se muito limitada para suprir as necessidades de serviços de saneamento. ${ }^{15,17}$

Referente aos hábitos alimentares, os idosos que relataram comer saladas, verduras e frutas tiveram um índice de infecção por enteroparasitos maior do que aqueles que se alimentaram com carnes, pães e massas. Provavelmente o consumo de alimentos crus facilita a contaminação por protozoários não patogênicos, que foram encontrados nessa pesquisa, considerando este um indicador de contaminação por material fecal. ${ }^{13,14}$

Quando se correlaciona idosos e enteroparasitoses, observa-se que não existe uma sintomatologia específica para este grupo. A manifestação clínica dessas patologias é variável e algumas vezes inespecífica. Geralmente, a sintomatologia é proporcional ao número de parasitos nos hospedeiros. ${ }^{18}$

Através dos funcionários dos lares geriátricos, percebemos uma maior prevalência de enteroparasitoses em relação aos idosos, havendo uma confirmação quanto ao maior índice 
encontrado nas mulheres, inclusive com biparasitismo. Esses profissionais são responsáveis pela higiene e cuidado dos idosos institucionalizados, podendo ser uma fonte de contaminação para os mais velhos. Direto de pessoa para pessoa, a transmissão ocorre principalmente em comunidades fechadas. ${ }^{4,19}$

A taxa de infecção por enteroparasitos foi anos ou mais, com diferença estatisticamente significativa, dados também observados em relação à menor escolaridade, sugerindo a importância de termos consciência de que pessoas desprovidas de estudo, em qualquer faixa etária, e que não cuidam de seus hábitos de higiene, tornam-se mais propensas à contaminação, principalmente de idosos que se encontram dependentes e com menos contato com o ambiente externo. ${ }^{20}$

Embora, em nossos achados a prevalência

correspondeu à contaminação por E. nana e E. coli, protozoários não patogênicos, obtivemos um resultado relevante porque através dele, evidenciamos as condições encontradas nestas regiões gaúchas. Mesmo que as condições de saneamento básico relatadas parecem não ser tão favoráveis, de forma geral a Região Serrana do Rio Grande do Sul possui boas condições socioeconômicas - área de grande concentração de indústrias moveleiras, por exemplo- e sanitárias quando comparadas com as demais regiões brasileiras. Por esse motivo que outros estudos relatam altas prevalências, ${ }^{21,22}$ confirmando que

as parasitoses, apresentam variações de acordo com cada região.9,23 Portanto, são necessários não só para medir o problema das altas taxas de morbidade associadas a essas parasitoses, bem como para gerar dados para o planejamento de ações governamentais e conscientização dos idosos e funcionários. Programas constantes em relação ao controle destas infecções proporcionarão melhora da qualidade de vida, no que se refere à questão da Saúde Pública. ${ }^{24,25}$

\section{CONCLUSÃO}

O envelhecimento populacional traz alguns desafios para sociedade, exigindo implantação e efetivação de políticas públicas sociais e de saúde condizentes com a realidade encontrada no Brasil e no Rio Grande do Sul. Nesse sentido, não podemos esquecer as mudanças importantes que existiram nos últimos anos, nas Instituições de Longa Permanência (ILPIs). Justamente, para tentar garantir atenção interdisciplinar, multiprofissional, adequada e voltada para promoção da saúde - correspondendo as exigências reguladas às instituições pelas fiscalizações competentes. Desta forma, poderemos assegurar uma atenção integral à saúde, prevenindo as infecções parasitárias - por exemplo, através de medidas sanitárias e educacionais. Mesmo que ainda tenhamos muito a melhorar, para que possamos de fato ofertar uma qualidade de vida digna, aos idosos e trabalhadores, dessas instituições.

AGRADECIMENTOS: Agradecemos ao Instituto de Geriatria e Gerontologia Biomédica da PUCRS, em especial ao LABGEMP (Laboratório de Bioquímica, Genética Molecular e Parasitologia), pelo apoio institucional para o desenvolvimento desse trabalho.

\section{REFERÊNCIAS}

1. Corteletti IA, Casara MB, Herédia VBM. Idoso Asilado um Estudo Gerontolócico. Bulla LC, Mediondo MZ: Velhice, Dependência e Vida Cotidiana Institucional. 2a edição. Caxias do Sul/RS: Educs; Porto Alegre: Edipucrs, 2010. p. 89-109. 2. Justino GO, Janebro DI, Ferreira AM, Nóbrega M de FF, Queiroz MSR. Identificação e Tratamento de Endoparasitoses em Pacientes da Terceira Idade [Internet]. FIEP Bulletin; 2010; 80 (Special Edition): Article II. [Acessado em 10 de maio 2013]. Disponível em http://www.fiepbulletin.net/index.php/fiepbulletin/article/view/1725

3. Ely LS, Engroff P, Lopes GT, Werlang M, Gomes I, De Cali GA. Prevalência de Enteroparasitos em Idosos. Revista Brasileira de Geriatria e Gerontologia. 2011; 14(4):637-646.

4. Girotto KG, Grama DF, Cunha MJR, Faria ESM, Limongi JE, Pinto RMC, et al. Prevalência e fatores de risco para infecção por protozoários intestinais em idosos residentes nas Instituições de Longa Permanência no Sudeste do Brasil. Revista do Instituto de Medicina Tropical. 2013 Jan/Fev; 55(1):19-24. 
5. Coelho VMA, Ferreira M do C, Lessa CSS, Pineli PP, Almeida VRG, Alves RC, et al. Parasitologia no Programa Renascer: uma trajetória de orientação e prevenção de parasitoses em idosos. Revista Interagir. 2009 Jan/Dez; 14:65-70.

6. De Carli GA. Parasitologia Clínica - seleção de métodos e técnicas de laboratório para o diagnóstico das parasitoses humanas. 2a edição. São Paulo: Atheneu, 2007; Seção 1- Capítulo 2, Exames Macroscópico e Microscópico da Amostra Fecal Fresca e Preservada.

7. Hoffman WA, Pons JA, Janer JL. The sedimentation concentration method in schistosomiasis mansoni. The Puerto Rico Journal of Public Health and Tropical Medicine. 1934; 9:281-298.

8. Baermann G. Eine einfache Methode zur Auffindung von Ankylostomun (Nematoden). In: Larven in Erdproben. Neded Geneesk Labt Welteverden Feestbundel, Batavia 1917. p.41-47.

9. Santos SA, Merlini LS. Prevalência de Enteroparasitoses na população do município de Maria Helena, Paraná. Revista Ciência \& Saúde Coletiva. 2010; 15(3):899-905.

10. Macedo HS. Prevalência de Parasitos e Comensais Intestinais em Crianças de escolas da rede Pública Municipal de Paracatu (MG). Revista Brasileira de Análises Clínicas. 2005; 37(4):209-213.

11. Polaro SHI, Fideralino JCT, Nunes PAO, Feitosa ES, Gonçalves LHT. Idosos residentes em Instituições de longa permanência para idosos da região metropolitana de Belém-PA. Revista Brasileira de Geriatria e Gerontologia. 2012; 15(4):777-784. 12. Shakya B, Rai SK, Singh A, Shresta A. Intestinal parasitosis among the elderly people in Kathmandu Valley. Nepal Medical College Journal. 2006; 8:243-7.

13. Santos RCV, Hoerlle JL, Aquino ARC, De Carli GA. Prevalência de enteroparasitoses em pacientes ambulatoriais do Hospital Divina Providência de Porto Alegre. Revista Brasileira de Análises Clínicas. 2004; 36(4):241-3.

14. Soares B, Cantos GA. Detecção de estruturas parasitárias em hortaliças comercializadas na cidade de Florianópolis, SC, Brasil. Revista Brasileira de Ciências Farmacêuticas. 2006, Jul/Set; 42(3).

15. Dal Maso RA. As cidades do RS despejam esgoto nos rios. Políticas Públicas, Porto Alegre. Revistas eletrônicas FEE. 2008; 36(3). [Acessado em 30 de outubro de 2013]. Disponível em http://revistas.fee.tche.br/index.php/indicadores/article/ view/2191/2596

16. Saneamento Básico. Bento Gonçalves torna-se referência em investimentos em saneamento na Serra [Internet]. 2013 [citado em 09/10/2013]. Disponível em: http://www.saneamentobasico.com.br/portal/index.php/tratamento-de-agua/ bento-goncalves-torna-se-referencia-em-investimentos-em-saneamento-na-serra/

17. Jornal VS. Sinos recebe $77 \%$ de esgoto não tratado em São Leopoldo diz Semae [Internet]. 2013 [citado em 09/10/2013]. Disponível: http://www.jornalvs.com.br/realidade/476753/sinos-recebe-77-de-esgoto-nao-tratado-em-saoleopoldodizsemae.html

18. Furtado LFV, Melo ACFL. Prevalência e aspectos epidemiológicos de enteroaparasitoses na população geronte de Parnaíba, Estado do Piauí. Revista Sociedade Brasileira de Medicina Tropical. 2011 Jul/Ago; 44(4):513-515.

19. Silva JO, Capuano DM, Takayanagui OM, Giacometti JR E. Enteroparasitoses e onicomicoses em manipuladores de alimentos do município de Ribeirão Preto, SP, Brasil. Revista Brasileira de Epidemiologia. 2005; 8(4):385-92.

20. Chaimowics F. A saúde dos idosos brasileiros às vésperas século XXI: problemas, projeções e alternativas. Revista de Saúde Pública. 1997; 3(2):184-200.

21. Hurtado-Guerrero AF, Alencar FH, Hurtaro-Guerreiro JC. Ocorrência de enteroparasitos na população geronte de Nova Olinda do Norte, Amazonas, Brasil. Revista Acta Amazônica. 2005; 35(4):487-490.

22. Ludwig KM, Frei F, Alvares Filho F, Paes-Ribeiro JT. Correlação entre condições de saneamento básico e parasitoses intestinais na população de Assis. Revista da Sociedade Brasileira de Medicina Tropical. 1999; 32(5):547-55.

23. Matos AS, Murai HC. Prevalência de parasitoses intestinais por Helmintos e Protozoários em idosos. Revista de Enfermagem UNISA. 2005; 6:9-14.

24. Andrade EC, Leite ICG, Rodrigues V de O, Cesca, MG. Parasitoses Intestinais: Uma revisão sobre seus aspectos sociais, epidemiológicos, clínicos e terapêuticos. Revista de APS. 2010 Abr/Jun 3(2):231-240.

25. Lima FF, Koivisto MB, Perri SHV, Bresciani KDS. O conhecimento de idosos sobre parasitoses em Instituições não governamentais no município de Araçatuba. Revista de Ciências Exatas. 2008; 4(1):77.

Recebido em: 02 de dezembro de 2014. Aprovado em: 06 de junho de 2015. 